\title{
Orthonormal bases with nonlinear phases
}

\author{
Tao Qian • Rui Wang • Yuesheng Xu • \\ Haizhang Zhang
}

Received: 6 July 2008 / Accepted: 5 February 2009

(C) Springer Science + Business Media, LLC 2009

\begin{abstract}
For adaptive representation of nonlinear signals, the bank $\mathcal{M}$ of real square integrable functions that have nonlinear phases and nonnegative instantaneous frequencies under the analytic signal method is investigated. A particular class of functions with explicit expressions in $\mathcal{M}$ is obtained using
\end{abstract}

Communicated by Qiyu Sun.

Supported in part by Macau Science and Technology Fund 051/2005/A.

Supported in part by the US National Science Foundation under grants CCR-0407476 and DMS-0712827, by National Aeronautics and Space Administration under Cooperative Agreement NNX07AC37A, by the Natural Science Foundation of China under grants 10371122 and 10631080, and by the Education Ministry of the People's Republic of China under the Changjiang Scholar Chair Professorship Program through Sun Yat-sen University.

\section{T. Qian}

Department of Mathematics, Faculty of Science and Technology, University of Macau,

Macau, People's Republic of China

e-mail: fsttq@umac.mo

R. Wang

School of Information Science and Engineering,

Graduate University of Chinese Academy of Sciences, Beijing 100190,

People's Republic of China

e-mail: wangrui@gucas.ac.cn

Y.Xu $(\varangle) \cdot$ H. Zhang

Department of Mathematics, Syracuse University, Syracuse, NY 13244, USA

e-mail: yxu06@syr.edu

H. Zhang

e-mail: hzhang12@syr.edu 
recent results on the Bedrosian identity. We then construct orthonormal bases for the Hilbert space of real square integrable functions with the basis functions from $\mathcal{M}$.

Keywords The Hilbert transform - The empirical mode decomposition • Time-frequency analysis $\cdot$ Orthonormal bases $\cdot$ Hardy spaces

Mathematics Subject Classifications (2000) 46E20 • 46A35

\section{Introduction}

The classical Fourier basis has been proven to be an efficient approach to represent a linear and stationary signal. However, it is not efficient to represent a nonlinear and nonstationary signal (cf., [11]). There were many methods $([4,6,11,13-15,19]$ and the references therein) proposed recently to overcome the shortcoming of the Fourier analysis from both empirical and mathematical points of view. In particular, the study of the instantaneous amplitude and frequency [11] of a nonlinear and nonstationary signal has attracted much attention. A common approach to define the instantaneous amplitude and frequency is through the Hilbert transform. It is desirable to construct orthonormal bases for the Hilbert space of real square integrable functions which have nonlinear phases and admit well-behaved Hilbert transforms. The goal of this paper is to present general constructions of such orthonormal bases.

We begin with recalling the definition of the Hilbert transform. The Fourier transform $\hat{f}$ of a function $f \in L^{1}(\mathbb{R})$ is defined by

$$
\hat{f}(\xi)=(\mathcal{F} f)(\xi):=\int_{\mathbb{R}} f(x) e^{-i x \xi} \mathrm{d} x, \quad \xi \in \mathbb{R} .
$$

We can extend the Fourier transform to a unitary operator ([7], page 20) on $L^{2}(\mathbb{R})$ through a standard approximation process [10]. The Hilbert transform $H$ is a bounded operator on $L^{2}(\mathbb{R})$ defined via the Fourier multiplier $-i$ sgn, where $\operatorname{sgn}(\xi)$ takes values $-1,0$ and 1 for $\xi<0, \xi=0$ and $\xi>0$, respectively (cf., [10]). Specifically, we have for each $f \in L^{2}(\mathbb{R})$ that

$$
(H f)^{\wedge}(\xi)=-i \operatorname{sgn}(\xi) \hat{f}(\xi), \quad \xi \in \mathbb{R} .
$$

Set $L_{\mathrm{r}}^{2}(\mathbb{R}):=\left\{f: f \in L^{2}(\mathbb{R}), f\right.$ is real-valued $\}$, which is a Hilbert space over $\mathbb{R}$. The analytic signal $A f$ of a function $f \in L_{\mathrm{r}}^{2}(\mathbb{R})$ is defined by

$$
A f:=f+i H f .
$$

It may be rewritten as

$$
(A f)(t)=\gamma(t) e^{i \phi(t)}, \quad t \in \mathbb{R}
$$


with $\gamma \geq 0$ and $\phi$ a real function. The above equation gives $f$ an amplitudefrequency modulation

$$
f(t)=\gamma(t) \cos \phi(t), \quad t \in \mathbb{R} .
$$

The values $\gamma(t)$ and $\phi(t)$ above are then considered as the instantaneous amplitude and phase of signal $f$ at time $t$, respectively. Note that the derivative $\phi^{\prime}$ taken as the instantaneous frequency of $f$ is physically meaningful only if it is nonnegative. We say that $f$ admits a well-behaved Hilbert transform if the derivative of $\phi$ in (1.2) is nonnegative.

In general, a real signal may not admit a well-behaved Hilbert transform. The empirical mode decomposition (EMD) introduced in [11] is a numerical algorithm aiming at decomposing a signal into a sum of signals each of which admits a well-behaved Hilbert transform. The analytic signal method can then be applied to each summand to yield a sound energy-frequency-time distribution. The EMD algorithm works well for many applications but at the same time it does not for some cases. It is desirable to build a solid mathematical base for the algorithm. There are two stages in building such a base for the useful algorithm, [21]. The first is to construct a large bank $\mathcal{M}$ of functions $f \in L_{r}^{2}(\mathbb{R})$ such that

$$
(A f)(t)=\gamma(t) e^{i \phi(t)}, \gamma(t) \geq 0, \phi^{\prime}(t) \geq 0, \quad t \in \mathbb{R} .
$$

The second is to establish an adaptive and rapid algorithm to decompose an arbitrary function $f \in L_{\mathrm{r}}^{2}(\mathbb{R})$ into a sum of functions in $\mathcal{M}$ with the summand decaying fast.

This paper serves as a first attempt to the above two stages. We simply aim at enlarging the existing class of functions with explicit expressions in $\mathcal{M}$, and providing a way of decomposing a square integrable function into a sum of functions in $\mathcal{M}$. We shall not emphasize the physical meaning of such a decomposition. Neither shall we discuss the decayness of components of the decomposition, which, however, deserves careful attention in the future. We intend to address the issue in another occasion.

The exposition of the paper is organized as follows. We first construct in Section 2 a class of functions with explicit expressions in $\mathcal{M}$ using recent developments [20,23] on the Bedrosian identity [2]. To decompose a function $f \in L_{\mathrm{r}}^{2}(\mathbb{R})$ into a sum of functions in $\mathcal{M}$, we construct orthonormal bases for the real Hilbert space $L_{\mathrm{r}}^{2}(\mathbb{R})$ with the basis functions in $\mathcal{M}$. Two constructions along with concrete examples are presented in Sections 3 and 4. Finally in Section 5, we give similar constructions of orthonormal bases for $L_{\mathrm{r}}^{2}[-\pi, \pi]$, the Hilbert space of real functions in $L^{2}[-\pi, \pi]$.

\section{Functions admitting a well-behaved Hilbert transform}

We construct in this section functions that admit a well-behaved Hilbert transform. An approach for constructing functions in $\mathcal{M}$ was proposed by the 
third author in 2002. It is to find nonnegative $\gamma \in L^{2}(\mathbb{R})$ and real $\phi \in C^{1}(\mathbb{R})$ satisfying the nonlinear singular integral equation

$$
[H(\gamma(\cdot) \cos \phi(\cdot))](t)=\gamma(t) \sin \phi(t), \quad t \in \mathbb{R}
$$

subjected to the constraint that

$$
\frac{\mathrm{d} \phi(t)}{\mathrm{d} t} \geq 0, \quad t \in \mathbb{R} .
$$

Motivated by this approach, we shall first consider an equation similar to (2.1) for periodic functions and then obtain functions in $\mathcal{M}$ through the Cayley transform. Let us make preparations for this by introducing the Hardy spaces $[8,9,18]$.

Set $\mathbb{U}:=\{z \in \mathbb{C}:|z|<1\} \quad, \quad \partial \mathbb{U}:=\{z \in \mathbb{C}:|z|=1\}$ and $\mathbb{C}_{+}:=\{z \in \mathbb{C}:$ $\operatorname{Im}(z)>0\}$. The set of all the holomorphic functions on $\mathbb{C}_{+}$and $\mathbb{U}$ is denoted by $\mathbf{H}\left(\mathbb{C}_{+}\right)$and $\mathbf{H}(\mathbb{U})$, respectively. We shall work on the Hardy spaces

$$
\begin{gathered}
\mathbf{H}^{2}\left(\mathbb{C}_{+}\right):=\left\{h \in \mathbf{H}\left(\mathbb{C}_{+}\right): \sup \left\{\int_{\mathbb{R}}|h(x+i y)|^{2} \mathrm{~d} x: y>0\right\}<\infty\right\}, \\
\mathbf{H}^{2}(\mathbb{U}):=\left\{h \in \mathbf{H}(\mathbb{U}): \sup \left\{\int_{-\pi}^{\pi}\left|h\left(r e^{i t}\right)\right|^{2} \mathrm{~d} t: r \in(0,1)\right\}<\infty\right\}, \\
\mathbf{H}^{\infty}\left(\mathbb{C}_{+}\right):=\left\{h \in \mathbf{H}\left(\mathbb{C}_{+}\right): \sup \left\{|h(z)|: z \in \mathbb{C}_{+}\right\}<\infty\right\}, \\
\mathbf{H}^{\infty}(\mathbb{U}):=\{h \in \mathbf{H}(\mathbb{U}): \sup \{|h(z)|: z \in \mathbb{U}\}<\infty\} .
\end{gathered}
$$

For a fixed $\alpha>0$, we introduce for each $t \in \mathbb{R}$ the cone in $\mathbb{C}_{+}$

$$
\Gamma_{\alpha}(t):=\left\{z=x+i y \in \mathbb{C}_{+}:|x-t|<\alpha y\right\} .
$$

For each $f \in \mathbf{H}^{p}\left(\mathbb{C}_{+}\right), p \in\{2, \infty\}$, there exists a $g \in L^{p}(\mathbb{R})$ such that for almost every $t \in \mathbb{R}$ there holds

$$
\lim _{\Gamma_{\alpha}(t) \ni z \rightarrow t} f(z)=g(t) .
$$

Likewise, if we set for a fixed $\varepsilon \in(0,1)$ and for every $\theta \in[-\pi, \pi]$

$$
\Omega_{\varepsilon}\left(e^{i \theta}\right):=\left\{\lambda e^{i \theta}+(1-\lambda) z: \lambda \in(0,1),|z|<\varepsilon\right\}
$$

then there exists for each $f \in \mathbf{H}^{p}(\mathbb{U}), p \in\{2, \infty\}$, a $g \in L^{p}(\partial \mathbb{U})$ such that for almost every $\theta \in[-\pi, \pi]$

$$
\lim _{\Omega_{\varepsilon}\left(e^{i \theta}\right) \ni z \rightarrow e^{i \theta}} f(z)=g\left(e^{i \theta}\right) .
$$

In both cases, we call the function $g$ the nontangential boundary limit of $f$, which is independent of the choice of the $\alpha>0$ or $\varepsilon \in(0,1)$. For simplicity, we shall use the same notation for a function in Hardy spaces as that for 
its nontangential boundary limit. The spaces $\mathbf{H}^{2}\left(\mathbb{C}_{+}\right)$and $\mathbf{H}^{2}(\mathbb{U})$ are Hilbert spaces endowed, respectively, with the inner products

$$
\langle f, g\rangle_{\mathbf{H}^{2}\left(\mathbb{C}_{+}\right)}:=\int_{\mathbb{R}} f(t) \overline{g(t)} \mathrm{d} t, \quad f, g \in \mathbf{H}^{2}\left(\mathbb{C}_{+}\right)
$$

and

$$
\langle f, g\rangle_{\mathbf{H}^{2}(\mathbb{U})}:=\frac{1}{2 \pi} \int_{-\pi}^{\pi} f\left(e^{i t}\right) \overline{g\left(e^{i t}\right)} \mathrm{d} t, \quad f, g \in \mathbf{H}^{2}(\mathbb{U}) .
$$

These two spaces are connected through the Cayley transform. The conformal mapping from $\mathbb{C}_{+}$to $\mathbb{U}$ defined by

$$
K(w):=\frac{i-w}{i+w}, \quad w \in \mathbb{C}_{+}
$$

is called the Cayley transform and it extends continuously as a bijective mapping from the extended real line to $\partial \mathbb{U}$. The correspondence between the boundaries is

$$
e^{i s}=K(t)=\frac{i-t}{i+t}, \quad t \in \mathbb{R}, s \in(-\pi, \pi),
$$

which implies

$$
s=2 \arctan t, \quad t \in \mathbb{R} .
$$

With the Cayley transform, the linear transformation $T$ from $\mathbf{H}^{2}(\mathbb{U})$ to $\mathbf{H}^{2}\left(\mathbb{C}_{+}\right)$ defined for $f \in \mathbf{H}^{2}(\mathbb{U})$ by

$$
T f:=\frac{1}{\sqrt{\pi}} \frac{1}{1-i z}(f \circ K)
$$

is an isomorphism ([7], page 19).

The Hilbert transform, as indicated by the analytic signal approach described in the introduction, is fundamental in the time-frequency analysis of square integrable signals. Its counterpart in the time-frequency analysis of periodic signals is the circular Hilbert transform $\tilde{H}$ defined for $f \in L^{2}[-\pi, \pi]$ by

$$
(\tilde{H} f)(t):=\sum_{k \in \mathbb{Z}}-i \operatorname{sgn}(k) c_{k}(f) e^{i k t}, \quad t \in[-\pi, \pi],
$$

where $c_{k}(f)$ is the $k$ th Fourier coefficient of $f$ defined by

$$
c_{k}(f):=\frac{1}{2 \pi} \int_{-\pi}^{\pi} f(t) e^{-i k t} \mathrm{~d} t .
$$

We wish to construct functions with explicit expressions in the bank $\tilde{\mathcal{M}}$ of functions $f \in L_{\mathrm{r}}^{2}[-\pi, \pi]$ such that

$$
(f+i \tilde{H} f)(t)=\rho(t) e^{i \theta(t)}, \rho(t) \geq 0, \theta^{\prime}(t) \geq 0, \quad t \in[-\pi, \pi]
$$


and orthonormal bases from $\tilde{\mathcal{M}}$ for $L_{\mathrm{r}}^{2}[-\pi, \pi]$. The latter question is postponed until Section 5. Functions in $\tilde{\mathcal{M}}$ may be obtained by finding nonnegative $\rho \in L^{2}[-\pi, \pi]$ and real $\theta \in C^{1}[-\pi, \pi]$ with a nonnegative derivative such that

$$
[\tilde{H}(\rho(\cdot) \cos \theta(\cdot))](t)=\rho(t) \sin \theta(t), \quad t \in[-\pi, \pi] .
$$

This section is devoted to constructing functions in $\mathcal{M}$ and $\tilde{\mathcal{M}}$ with explicit expressions. The construction method is to solve (2.1), (2.8) with a prescribed phase function.

Let $\mathbb{N}$ be the set of all the positive integers and $\mathbb{Z}_{+}:=\mathbb{N} \cup\{0\}$. To enumerate finite sets, we define for each $n \in \mathbb{N}, \mathbb{N}_{n}:=\{1,2, \ldots, n\}$ and $\mathbb{Z}_{n}:=\{0,1, \ldots$, $n-1$ \}. The first problem we consider is that given the phase function $\theta$ in (2.8) that satisfies the condition

$$
e^{i \theta(t)}=\prod_{j \in \mathbb{N}_{n}} \frac{e^{i t}-\lambda_{j}}{1-\lambda_{j} e^{i t}}, \quad t \in[-\pi, \pi],
$$

where $n \in \mathbb{N}, \lambda_{j} \in[0,1), j \in \mathbb{N}_{n}$, we solve (2.8) for amplitudes $\rho$. It can be verified that the derivative of $\theta$ given above is positive, [15-17]. We present the answer to this problem in the next theorem. To this end, we recall the wellknown Cauchy integral formula ([1], page 119) that if $f$ is analytic on an open subset of $\mathbb{C}$ that contains $\mathbb{U} \cup \partial \mathbb{U}$ then there holds for each $z_{0} \in \mathbb{U}$

$$
f\left(z_{0}\right)=\frac{1}{2 \pi i} \int_{\partial \mathbb{U}} \frac{f(z)}{z-z_{0}} d z .
$$

Theorem 2.1 Let $n \in \mathbb{N}, \lambda_{j} \in[0,1), j \in \mathbb{N}_{n}$, and $\theta$ be determined by (2.9). Then a real function $\rho \in L^{2}[-\pi, \pi]$ satisfies (2.8) if and only if there exists $b_{j} \in \mathbb{C}$, $j \in \mathbb{Z}_{n-1}$ and $b_{n-1}, c \in \mathbb{R}$, such that

$$
\rho(t)=\operatorname{Re}\left(\frac{e^{i t} \sum_{j \in \mathbb{Z}_{n}} b_{j} e^{i j t}}{\prod_{j \in \mathbb{N}_{n}}\left(1-\lambda_{j} e^{i t}\right)}\right)+c, \quad t \in[-\pi, \pi] .
$$

Proof Since by (2.6), $\theta$ given in (2.9) satisfies that $\tilde{H} \cos \theta=\sin \theta, \rho \in$ $L^{2}[-\pi, \pi]$ satisfies $(2.8)$ if and only if

$$
\tilde{H}(\rho(\cdot) \cos \theta(\cdot))(t)=\rho(t) \tilde{H}(\cos \theta(\cdot))(t), \quad t \in[-\pi, \pi] .
$$

This is a circular Bedrosian identity studied in $[17,23]$. To make use of the results there, we set $g:=\cos \theta$ and assume that there holds for some $\left[\alpha_{j}: j \in\right.$ $\left.\mathbb{Z}_{+}\right] \in \ell^{2}\left(\mathbb{Z}_{+}\right)$that

$$
e^{i \theta(t)}=\sum_{j \in \mathbb{Z}_{+}} \alpha_{j} e^{i j t}, \quad t \in[-\pi, \pi]
$$

Since $\lambda_{j}$ is real for each $j \in \mathbb{N}_{n}$, we observe from (2.9) that $\alpha_{j} \in \mathbb{R}, j \in \mathbb{Z}_{+}$. 
By a necessary and sufficient condition for circular Bedrosian identities established in [23], (2.12) holds if and only if the following three equations are satisfied

$$
\begin{gathered}
\sum_{j \in \mathbb{N}} c_{j}(\rho) c_{-j}(g)=\sum_{j \in \mathbb{N}} c_{-j}(\rho) c_{j}(g), \\
c_{k}(\rho) c_{0}(g)+2 \sum_{j \in \mathbb{N}} c_{k+j}(\rho) c_{-j}(g)=0, \quad k \in \mathbb{N}, \\
c_{-k}(\rho) c_{0}(g)+2 \sum_{j \in \mathbb{N}} c_{-k-j}(\rho) c_{j}(g)=0, \quad k \in \mathbb{N} .
\end{gathered}
$$

Noting that $\rho$ is real and $\alpha_{j} \in \mathbb{R}, j \in \mathbb{Z}_{+}$, we have that

$$
c_{-j}(\rho)=\overline{c_{j}(\rho)}, c_{0}(g)=\alpha_{0}, c_{j}(g)=c_{-j}(g)=\frac{1}{2} c_{j}\left(e^{i \theta}\right)=\frac{1}{2} \alpha_{j}, \quad j \in \mathbb{N} .
$$

The above relations imply that (2.13), (2.14) and (2.15) are of the forms

$$
\begin{gathered}
\sum_{j \in \mathbb{N}}\left(c_{j}(\rho)-c_{-j}(\rho)\right) \alpha_{j}=0, \\
c_{k}(\rho) \alpha_{0}+\sum_{j \in \mathbb{N}} c_{k+j}(\rho) \alpha_{j}=0, \quad k \in \mathbb{N}, \\
c_{-k}(\rho) \alpha_{0}+\sum_{j \in \mathbb{N}} c_{-k-j}(\rho) \alpha_{j}=0, \quad k \in \mathbb{N},
\end{gathered}
$$

where (2.17) and (2.18) are equivalent. Thus, we get that $\rho \in L^{2}[-\pi, \pi]$ satisfies (2.8) if and only if there holds (2.16) and (2.17).

By the Parseval identity for $L^{2}[-\pi, \pi],(2.17)$ is equivalent to that

$$
\int_{-\pi}^{\pi}\left(\sum_{j \in \mathbb{Z}_{+}} c_{j+1}(\rho) e^{i j t}\right) \frac{e^{i k t} e^{i \theta(t)}}{\mathrm{d} t}=0, \quad k \in \mathbb{Z}_{+},
$$

that is,

$$
\int_{-\pi}^{\pi}\left(\sum_{j \in \mathbb{Z}_{+}} c_{j+1}(\rho) e^{i j t}\right) e^{-i k t} \prod_{j \in \mathbb{N}_{n}} \frac{1-\lambda_{j} e^{i t}}{e^{i t}-\lambda_{j}} \mathrm{~d} t=0, \quad k \in \mathbb{Z}_{+} .
$$

Equation 2.19 holds if and only if there exists $\left[c_{j}: j \in \mathbb{N}\right] \in \ell^{2}(\mathbb{N})$ such that

$$
\left(\sum_{j \in \mathbb{Z}_{+}} c_{j+1}(\rho) e^{i j t}\right) \prod_{j \in \mathbb{N}_{n}}\left(1-\lambda_{j} e^{i t}\right)=\left(\sum_{j \in \mathbb{N}} c_{j} e^{-i j t}\right) \prod_{j \in \mathbb{N}_{n}}\left(e^{i t}-\lambda_{j}\right), \quad t \in[-\pi, \pi] .
$$


The highest order of the trigonometric series on the right hand side of (2.20) is $n-1$, while the lowest order of the trigonometric series on the left is 0 . Consequently, the trigonometric function series expressed by both sides must have orders 0 to $n-1$ only. Therefore, if (2.20) holds then there exists $b_{j} \in \mathbb{C}$, $j \in \mathbb{Z}_{n}$, such that

$$
\left(\sum_{j \in \mathbb{Z}_{+}} c_{j+1}(\rho) e^{i j t}\right) \prod_{j \in \mathbb{N}_{n}}\left(1-\lambda_{j} e^{i t}\right)=\sum_{j \in \mathbb{Z}_{n}} b_{j} e^{i j t}, \quad t \in[-\pi, \pi] .
$$

Conversely, if (2.21) holds for some $b_{j} \in \mathbb{C}, j \in \mathbb{Z}_{n}$ then (2.20) is true for some $\left[c_{j}: j \in \mathbb{N}\right] \in \ell^{2}(\mathbb{N})$. We hence conclude that (2.17) holds if and only if there exists $b_{j} \in \mathbb{C}, j \in \mathbb{Z}_{n}$, such that (2.21) holds.

Suppose that $\rho \in L_{\mathrm{r}}^{2}[-\pi, \pi]$ satisfies (2.8). By the discussions above, there holds (2.16) and (2.21) for some $b_{j} \in \mathbb{C}, j \in \mathbb{Z}_{n}$. Since $\rho$ is real, we obtain from (2.21) that

$$
\rho(t)=2 \operatorname{Re}\left(\frac{e^{i t} \sum_{j \in \mathbb{Z}_{n}} b_{j} e^{i j t}}{\prod_{j \in \mathbb{N}_{n}}\left(1-\lambda_{j} e^{i t}\right)}\right)+c_{0}(\rho), \quad t \in[-\pi, \pi] .
$$

Using the above expression of $\rho$, we conclude that (2.16) is of the form

$$
\int_{-\pi}^{\pi} \frac{\sum_{j \in \mathbb{Z}_{n}} \operatorname{Im}\left(b_{j}\right) e^{i(n-j-1) t}}{\prod_{j \in \mathbb{N}_{n}}\left(1-\lambda_{j} e^{i t}\right)} \mathrm{d} t=0 .
$$

Through a change of variables, the above equation can be written as

$$
\int_{\partial \mathbb{U}} \frac{\sum_{j \in \mathbb{Z}_{n}} \operatorname{Im}\left(b_{j}\right) z^{n-1-j}}{z \prod_{j \in \mathbb{N}_{n}}\left(1-\lambda_{j} z\right)} d z=0 .
$$

By the Cauchy integral formula (2.10), we have that $\operatorname{Im}\left(b_{n-1}\right)=0$.

On the other hand, if $\rho$ has the form (2.11) for $b_{j} \in \mathbb{C}, j \in \mathbb{Z}_{n-1}$ and $b_{n-1}, c \in$ $\mathbb{R}$, then we have that

$$
\sum_{j \in \mathbb{Z}_{+}} c_{j+1}(\rho) e^{i j t}=\frac{\frac{1}{2} \sum_{j \in \mathbb{Z}_{n}} b_{j} e^{i j t}}{\prod_{j \in \mathbb{N}_{n}}\left(1-\lambda_{j} e^{i t}\right)}, \quad t \in[-\pi, \pi],
$$

which implies by the equivalence of (2.21) and (2.17) that (2.17) is satisfied. Equation 2.22 then follows from the Cauchy integral formula and $b_{n-1}$ being real. Together with (2.23) and the Parseval identity for $L^{2}[-\pi, \pi]$, (2.22) leads to (2.16). Consequently, we conclude that $\rho$ satisfies (2.8).

One can always choose big enough $c$ in (2.11) so that $\rho$ given there is nonnegative. We conclude in this case that $\rho \cos \theta$ for $\rho$ and $\theta$ so chosen is contained in $\tilde{\mathcal{M}}$. 
We next construct functions in $\mathcal{M}$ with explicit expressions. In this case, we choose $\phi$ to satisfy the equation

$$
e^{i \phi(t)}=\frac{1+i t}{\sqrt{1+t^{2}}} \prod_{j \in \mathbb{N}_{n}} \frac{e^{i 2 \arctan t}-\lambda_{j}}{1-\lambda_{j} e^{i 2 \arctan t}}, \quad t \in \mathbb{R},
$$

where $n \in \mathbb{N}$ and $\lambda_{j} \in[0,1), j \in \mathbb{N}_{n}$. It can be verified directly that $\phi$ defined above satisfies (2.2). Our next task is to construct $\gamma$ that satisfies (2.1) given $\phi$ in (2.24). Along this line, we need the following characterization of nontangential boundary limits of functions in $\mathbf{H}^{2}\left(\mathbb{C}_{+}\right)$(see, for example, [9], page 88 and [16]).

Lemma 2.2 Functions $f, g \in L_{r}^{2}(\mathbb{R})$ satisfies the equation $H f=g$ if and only if $f+i g$ is the nontangential boundary limit of some function in $\mathbf{H}^{2}\left(\mathbb{C}_{+}\right)$.

A similar result holds for the space $\mathbf{H}^{2}(\mathbb{U})$ (see, for example, $[9,18]$ ).

Lemma 2.3 Let $f \in L^{2}[-\pi, \pi]$. Then there exists a nontangential boundary limit $h$ of some function in $\mathbf{H}^{2}(\mathbb{U})$ such that $f=h\left(e^{i \cdot}\right)$ if and only if $c_{-j}(f)=0$ for all $j \in \mathbb{N}$.

We are now ready to present a construction of $\gamma$.

Theorem 2.4 Let $n \in \mathbb{N}$ and $\phi$ be given by (2.24) with $\lambda_{j} \in[0,1), j \in \mathbb{N}_{n}$. Then a real function $\gamma \in L^{2}(\mathbb{R})$ satisfies (2.1) if and only if there exists $b_{j} \in \mathbb{C}, j \in \mathbb{N}_{n}$ and $c \in \mathbb{R}$ such that

$$
\gamma(t)=\frac{1}{\sqrt{1+t^{2}}}\left(\operatorname{Re}\left(\frac{\sum_{j \in \mathbb{N}_{n}} b_{j} e^{i 2 j \arctan t}}{\prod_{j \in \mathbb{N}_{n}}\left(1-\lambda_{j} e^{i 2 \arctan t}\right)}\right)+c\right), t \in \mathbb{R} .
$$

Proof Let $\phi$ be a real function on $\mathbb{R}$ as described in the assumption. We set for each function $\gamma$ on $\mathbb{R}$

$$
\rho:=\left(\sqrt{1+t^{2}} \gamma\right) \circ \tan \left(\frac{\dot{-}}{2}\right) .
$$

One can see that $\gamma \in L^{2}(\mathbb{R})$ if and only if $\rho \in L^{2}[-\pi, \pi]$. Note also that there holds

$$
\left[\left(\gamma e^{i \phi}(1-i t)\right) \circ K^{-1}\right]\left(e^{i s}\right)=\rho(s) e^{i \theta(s)}, s \in(-\pi, \pi),
$$

where $\theta$ is defined by (2.9).

Let $\gamma \in L_{\mathrm{r}}^{2}(\mathbb{R})$. Denote by $\mathcal{B}\left(\mathbb{C}_{+}\right)$and $\mathcal{B}(\mathbb{U})$ the set of nontangential boundary limits of functions in $\mathbf{H}^{2}\left(\mathbb{C}_{+}\right)$and $\mathbf{H}^{2}(\mathbb{U})$, respectively. We claim that $\gamma e^{i \phi} \in \mathcal{B}\left(\mathbb{C}_{+}\right)$if and only if there exists an $h \in \mathcal{B}(\mathbb{U})$ such that

$$
h\left(e^{i \cdot}\right)=\rho e^{i \theta} .
$$

Firstly, if $\gamma e^{i \phi} \in \mathcal{B}\left(\mathbb{C}_{+}\right)$then by the isomorphism $(2.5), h:=\left(\gamma e^{i \phi}(1-i t)\right) \circ$ $K^{-1} \in \mathcal{B}(\mathbb{U})$ and it satisfies (2.28) by (2.27). On the other hand, suppose we 
have an $h \in \mathcal{B}(\mathbb{U})$ that satisfies (2.28). Still by the isomorphism (2.5), $\frac{h \circ K}{1-i t} \in$ $\mathcal{B}\left(\mathbb{C}_{+}\right)$. We obtain by (2.27) and (2.28) that

$$
\gamma(t) e^{i \phi(t)}=\frac{(h \circ K)(t)}{1-i t}, \quad t \in \mathbb{R}
$$

which proves that $\gamma e^{i \phi}$ is contained in $\mathcal{B}\left(\mathbb{C}_{+}\right)$.

It follows by the above claim, Lemmas 2.2 and 2.3 that $\gamma$ satisfies (2.1) if and only if there exists $\left[\alpha_{j}: j \in \mathbb{Z}_{+}\right] \in \ell^{2}\left(\mathbb{Z}_{+}\right)$such that

$$
\rho(s) e^{i \theta(s)}=\left(\sum_{j \in \mathbb{Z}} c_{j}(\rho) e^{i j s}\right) \prod_{j \in \mathbb{N}_{n}} \frac{e^{i s}-\lambda_{j}}{1-\lambda_{j} e^{i s}}=\sum_{j \in \mathbb{Z}_{+}} \alpha_{j} e^{i j s}, \quad s \in[-\pi, \pi] .
$$

By the definition (2.26), $\gamma$ is of the form (2.25) if and only if $\rho$ has the following form

$$
\rho(s)=\operatorname{Re}\left(\frac{\sum_{j \in \mathbb{N}_{n}} b_{j} e^{i j s}}{\prod_{j \in \mathbb{N}_{n}}\left(1-\lambda_{j} e^{i s}\right)}\right)+c, \quad s \in[-\pi, \pi] .
$$

We conclude that to prove the theorem it suffices to prove that (2.29) holds for some $\left[\alpha_{j}: j \in \mathbb{Z}_{+}\right] \in \ell^{2}\left(\mathbb{Z}_{+}\right)$if and only if (2.30) holds for some $b_{j} \in \mathbb{C}, j \in \mathbb{N}_{n}$ and $c \in \mathbb{R}$.

Suppose first that (2.30) holds for some $b_{j} \in \mathbb{C}, j \in \mathbb{N}_{n}$ and $c \in \mathbb{R}$. Then through a direct computation, we get that

$$
\begin{aligned}
\rho(s) e^{i \theta(s)}= & \left(\frac{\sum_{j \in \mathbb{N}_{n}} b_{j} e^{i j s}}{2 \prod_{j \in \mathbb{N}_{n}}\left(1-\lambda_{j} e^{i s}\right)}+c\right) \prod_{j \in \mathbb{N}_{n}} \frac{e^{i s}-\lambda_{j}}{1-\lambda_{j} e^{i s}} \\
& +\frac{\sum_{j \in \mathbb{N}_{n}} \bar{b}_{j} e^{i(n-j) s}}{2 \prod_{j \in \mathbb{N}_{n}}\left(1-\lambda_{j} e^{i s}\right)}, \quad s \in[-\pi, \pi] .
\end{aligned}
$$

It is clear that the above equation implies that (2.29) holds for some $\left[\alpha_{j}: j \in\right.$ $\left.\mathbb{Z}_{+}\right] \in \ell^{2}\left(\mathbb{Z}_{+}\right)$. Conversely, if (2.29) holds for some $\left[\alpha_{j}: j \in \mathbb{Z}_{+}\right] \in \ell^{2}\left(\mathbb{Z}_{+}\right)$then there exists $\left[\alpha_{j}^{\prime}: j \in \mathbb{Z}_{+}\right] \in \ell^{2}\left(\mathbb{Z}_{+}\right)$such that

$$
\left(\sum_{j \in \mathbb{N}} c_{-j}(\rho) e^{-i j s}\right) \prod_{j \in \mathbb{N}_{n}} \frac{e^{i s}-\lambda_{j}}{1-\lambda_{j} e^{i s}}=\sum_{j \in \mathbb{Z}_{+}} \alpha_{j}^{\prime} e^{i j s}, \quad s \in[-\pi, \pi] .
$$

The same reasoning as that used in the proof of Theorem 2.1 then yields by (2.31) that there exists $b_{j}^{\prime} \in \mathbb{C}, j \in \mathbb{Z}_{n}$ such that

$$
\sum_{j \in \mathbb{N}} c_{-j}(\rho) e^{-i j s}=\frac{\sum_{j \in \mathbb{Z}_{n}} b_{j}^{\prime} e^{i j s}}{\prod_{j \in \mathbb{N}_{n}}\left(e^{i s}-\lambda_{j}\right)}, \quad s \in[-\pi, \pi] .
$$

Noting that (2.32) implies that $\rho$ has the form (2.30) for some $b_{j} \in \mathbb{C}, j \in \mathbb{N}_{n}$ and $c \in \mathbb{R}$ completes the proof. 


\section{Orthonormal bases for $L_{r}^{2}(\mathbb{R})$}

We present two constructions of orthonormal bases for $L_{\mathrm{r}}^{2}(\mathbb{R})$ with the basis functions in $\mathcal{M}$. Our first result below shows that this task can be reduced to a construction of orthonormal bases for the Hardy space $\mathbf{H}^{2}\left(\mathbb{C}_{+}\right)$.

Theorem 3.1 Functions $M_{j} \in \mathbf{H}^{2}\left(\mathbb{C}_{+}\right), j \in \mathbb{Z}_{+}$, with nontangential boundary limits

$$
M_{j}(t)=\rho_{j}(t) e^{i \theta_{j}(t)}, \quad t \in \mathbb{R}, \quad j \in \mathbb{Z}_{+}
$$

form an orthonormal basis for $\mathbf{H}^{2}\left(\mathbb{C}_{+}\right)$if and only if $\sqrt{2} \rho_{j} \cos \theta_{j}, \sqrt{2} \rho_{j} \sin \theta_{j}$, $j \in \mathbb{Z}_{+}$, satisfy

$$
H\left(\rho_{j}(\cdot) \cos \theta_{j}(\cdot)\right)(t)=\rho_{j}(t) \sin \theta_{j}(t), t \in \mathbb{R}, \quad j \in \mathbb{Z}_{+}
$$

and constitute an orthonormal basis for $L_{r}^{2}(\mathbb{R})$.

Proof Suppose that $M_{j}, j \in \mathbb{Z}_{+}$, with the amplitude-phase modulation (3.1), form an orthonormal basis for $\mathbf{H}^{2}\left(\mathbb{C}_{+}\right)$. Let $f$ be an arbitrary function in $L_{\mathrm{r}}^{2}(\mathbb{R})$. By Lemma 2.2, $f+i H f$ is the nontangential boundary limit of some function in $\mathbf{H}^{2}\left(\mathbb{C}_{+}\right)$. There hence exists $\left[\alpha_{j}: j \in \mathbb{Z}_{+}\right] \in \ell^{2}\left(\mathbb{Z}_{+}\right)$such that

$$
f(t)+i(H f)(t)=\sum_{j \in \mathbb{Z}_{+}} \alpha_{j} M_{j}(t), \quad t \in \mathbb{R},
$$

where the equality holds in $L^{2}(\mathbb{R})$. The above equation implies that the linear span of $\mathcal{A}:=\left\{\sqrt{2} \rho_{j} \cos \theta_{j}, \sqrt{2} \rho_{j} \sin \theta_{j}: j \in \mathbb{Z}_{+}\right\}$is dense in $L_{\mathrm{r}}^{2}(\mathbb{R})$. It remains to prove the orthonormality of $\mathcal{A}$. To this end, we note that since $\rho_{j} e^{i \theta_{j}}$ is the nontangential boundary limit of $M_{j} \in \mathbf{H}^{2}\left(\mathbb{C}_{+}\right)$there holds for each $j \in \mathbb{Z}_{+}$that $H\left(\rho_{j}(\cdot) \cos \theta_{j}(\cdot)\right)(t)=\rho_{j}(t) \sin \theta_{j}(t), \quad H\left(\rho_{j}(\cdot) \sin \theta_{j}(\cdot)\right)(t)=-\rho_{j}(t) \cos \theta_{j}(t), t \in \mathbb{R}$.

By (1.1), we obtain that

$$
\mathcal{F}\left(\rho_{j} \sin \theta_{j}\right)(\xi)=-i \operatorname{sgn}(\xi) \mathcal{F}\left(\rho_{j} \cos \theta_{j}\right)(\xi), \quad \xi \in \mathbb{R} .
$$

Consequently, there holds for each $j, k \in \mathbb{Z}_{+}$

$$
\begin{aligned}
\left\langle M_{j}, M_{k}\right\rangle_{\mathbf{H}^{2}\left(\mathbb{C}_{+}\right)} & =\int_{\mathbb{R}} \mathcal{F}\left(\rho_{j} e^{i \theta_{j}}\right)(\xi) \overline{\mathcal{F}\left(\rho_{k} e^{i \theta_{k}}\right)(\xi)} d \xi \\
& =4 \int_{0}^{\infty} \mathcal{F}\left(\rho_{j} \cos \theta_{j}\right)(\xi) \overline{\mathcal{F}\left(\rho_{k} \cos \theta_{k}\right)(\xi)} d \xi .
\end{aligned}
$$

By the assumption that $M_{j}, j \in \mathbb{Z}_{+}$, form an orthonormal basis for $\mathbf{H}^{2}\left(\mathbb{C}_{+}\right)$, we get

$$
\int_{0}^{\infty} \mathcal{F}\left(\rho_{j} \cos \theta_{j}\right)(\xi) \overline{\mathcal{F}\left(\rho_{k} \cos \theta_{k}\right)(\xi)} d \xi=\frac{1}{4} \delta_{j, k}
$$


where $\delta_{j, k}$ denotes the Kronecker delta. Finally, we calculate by the basic properties of the Fourier transform and (3.3) that

$$
\begin{aligned}
\left\langle\rho_{j} \cos \theta_{j}, \rho_{k} \cos \theta_{k}\right\rangle_{L^{2}(\mathbb{R})} & =\left\langle\rho_{j} \sin \theta_{j}, \rho_{k} \sin \theta_{k}\right\rangle_{L^{2}(\mathbb{R})} \\
& =2 \operatorname{Re}\left(\int_{0}^{\infty} \mathcal{F}\left(\rho_{j} \cos \theta_{j}\right)(\xi) \overline{\mathcal{F}\left(\rho_{k} \cos \theta_{k}\right)(\xi)} d \xi\right)
\end{aligned}
$$

and

$$
\left\langle\rho_{j} \cos \theta_{j}, \rho_{k} \sin \theta_{k}\right\rangle_{L^{2}(\mathbb{R})}=-2 \operatorname{Im}\left(\int_{0}^{\infty} \mathcal{F}\left(\rho_{j} \cos \theta_{j}\right)(\xi) \overline{\mathcal{F}\left(\rho_{k} \cos \theta_{k}\right)(\xi)} d \xi\right) .
$$

The above two equations together with (3.4) prove the orthonormality of $\mathcal{A}$ in $L_{\mathrm{r}}^{2}(\mathbb{R})$.

Conversely, assume that $\sqrt{2} \rho_{j} \cos \theta_{j}, \sqrt{2} \rho_{j} \sin \theta_{j}, j \in \mathbb{Z}_{+}$, satisfy (3.2) for all $j \in \mathbb{Z}_{+}$and constitute an orthonormal basis for $L_{r}^{2}(\mathbb{R})$. It follows by Lemma 2.2 that $M_{j} \in \mathbf{H}^{2}\left(\mathbb{C}_{+}\right), j \in \mathbb{Z}_{+}$. It is clear that there holds for each $j, k \in \mathbb{Z}_{+}$

$$
\left\langle M_{j}, M_{k}\right\rangle_{\mathbf{H}^{2}\left(\mathbb{C}_{+}\right)}=\left\langle\rho_{j} \cos \theta_{j}, \rho_{k} \cos \theta_{k}\right\rangle_{L^{2}(\mathbb{R})}+\left\langle\rho_{j} \sin \theta_{j}, \rho_{k} \sin \theta_{k}\right\rangle_{L^{2}(\mathbb{R})}=\delta_{j, k}
$$

which confirms the orthonormality of $\left\{M_{j}: j \in \mathbb{Z}_{+}\right\}$in $\mathbf{H}^{2}\left(\mathbb{C}_{+}\right)$. It suffices to prove that the linear span of $\left\{M_{j}: j \in \mathbb{Z}_{+}\right\}$is dense in $\mathbf{H}^{2}\left(\mathbb{C}_{+}\right)$. Set $M \in \mathbf{H}^{2}\left(\mathbb{C}_{+}\right)$. Suppose that its nontangential boundary limit has the form

$$
M(t)=\rho(t) e^{i \theta(t)}, \quad t \in \mathbb{R},
$$

where $\rho \geq 0$ and $\theta$ is real. We can find $\left[\alpha_{j}: j \in \mathbb{Z}_{+}\right],\left[\beta_{j}: j \in \mathbb{Z}_{+}\right] \in \ell^{2}\left(\mathbb{Z}_{+}\right)$such that there holds the equality in $L^{2}(\mathbb{R})$

$$
\rho(t) \cos \theta(t)=\sum_{j \in \mathbb{Z}_{+}} \alpha_{j} \rho_{j}(t) \cos \theta_{j}(t)+\sum_{j \in \mathbb{Z}_{+}} \beta_{j} \rho_{j}(t) \sin \theta_{j}(t), t \in \mathbb{R} .
$$

Applying the Hilbert transform to both sides of the above equation yields that

$$
\rho(t) \sin \theta(t)=\sum_{j \in \mathbb{Z}_{+}} \alpha_{j} \rho_{j}(t) \sin \theta_{j}(t)-\sum_{j \in \mathbb{Z}_{+}} \beta_{j} \rho_{j}(t) \cos \theta_{j}(t), t \in \mathbb{R},
$$

where the equation also holds in $L^{2}(\mathbb{R})$. Combining (3.5) and (3.6) follows that there holds in $L^{2}(\mathbb{R})$

$$
M(t)=\sum_{j \in \mathbb{Z}_{+}}\left(\alpha_{j}-i \beta_{j}\right) \rho_{j}(t) e^{i \theta_{j}(t)} .
$$

This equation implies that the linear span of $\left\{M_{j}: j \in \mathbb{Z}_{+}\right\}$is dense in $\mathbf{H}^{2}\left(\mathbb{C}_{+}\right)$ and proves the theorem.

Motivated by Theorem 3.1, we next consider the construction of orthonormal bases $\left\{M_{j}: j \in \mathbb{Z}_{+}\right\}$for $\mathbf{H}^{2}\left(\mathbb{C}_{+}\right)$. Our first construction makes use of the outer functions in $\mathbf{H}^{2}\left(\mathbb{C}_{+}\right)$. Those are functions $h \in \mathbf{H}\left(\mathbb{C}_{+}\right)$of the form

$$
h(z)=\exp \{u(z)+i v(z)\}, \quad z \in \mathbb{C}_{+},
$$


where

$$
u(z):=\frac{1}{\pi} \int_{\mathbb{R}} \frac{y}{(x-t)^{2}+y^{2}}(\log \varphi)(t) \mathrm{d} t, \quad z=x+i y \in \mathbb{C}_{+}
$$

with $\varphi$ being a nonnegative function such that

$$
\int_{R} \frac{|(\log \varphi)(t)| \mathrm{d} t}{1+t^{2}}<\infty
$$

and where $v$ is a harmonic conjugate function of $u$, [9]. A function $f \in \mathbf{H}^{2}\left(\mathbb{C}_{+}\right)$ is an outer function if and only if the linear span

$$
\operatorname{span}\left\{f(\cdot) e^{i y \cdot}: y \geq 0\right\}
$$

is dense in $\mathbf{H}^{2}\left(\mathbb{C}_{+}\right)$, [12]. There is another characterization of outer functions in $\mathbf{H}^{2}\left(\mathbb{C}_{+}\right)$, [9]. It states that $f \in \mathbf{H}^{2}\left(\mathbb{C}_{+}\right)$is an outer function if and only if there holds

$$
\log |f(i)|=\frac{1}{\pi} \int_{\mathbb{R}} \frac{\log |f(t)|}{1+t^{2}} \mathrm{~d} t .
$$

For an $f \in \mathbf{H}^{\infty}\left(\mathbb{C}_{+}\right)$we denote by $\mathbf{H}_{f}^{2}\left(\mathbb{C}_{+}\right)$the Hilbert space completed upon the linear space of functions in $\mathbf{H}^{2}\left(\mathbb{C}_{+}\right)$under the inner product

$$
\langle g, h\rangle_{\mathbf{H}_{f}^{2}\left(\mathbb{C}_{+}\right)}:=\int_{\mathbb{R}} g(t) \overline{h(t)}|f(t)|^{2} \mathrm{~d} t, \quad g, h \in \mathbf{H}^{2}\left(\mathbb{C}_{+}\right) .
$$

Theorem 3.2 Suppose that $f_{1}, f_{2} \in \mathbf{H}^{2}\left(\mathbb{C}_{+}\right)$satisfy that $f_{1} / f_{2} \in \mathbf{H}^{\infty}\left(\mathbb{C}_{+}\right)$and $f_{1}$ is an outer function. If $e_{j} \in \mathbf{H}^{2}\left(\mathbb{C}_{+}\right), j \in \mathbb{Z}_{+}$, form an orthonormal basis for $\mathbf{H}_{f_{1} / f_{2}}^{2}\left(\mathbb{C}_{+}\right)$, then $\frac{f_{1}}{f_{2}} e_{j}, j \in \mathbb{Z}_{+}$, form an orthonormal basis for $\mathbf{H}^{2}\left(\mathbb{C}_{+}\right)$.

Proof Suppose that all the assumptions are satisfied. We first see that $\frac{f_{1}}{f_{2}} e \in$ $\mathbf{H}^{2}\left(\mathbb{C}_{+}\right)$whenever $e \in \mathbf{H}^{2}\left(\mathbb{C}_{+}\right)$. This observation together with the definition (3.8) ensures immediately that $\frac{f_{1}}{f_{2}} e_{j}, j \in \mathbb{Z}_{+}$, form an orthonormal sequence in $\mathbf{H}^{2}\left(\mathbb{C}_{+}\right)$. It remains to show that their linear span is dense in $\mathbf{H}^{2}\left(\mathbb{C}_{+}\right)$. Set $y \in[0, \infty)$. By the assumption that $e_{j}, j \in \mathbb{Z}_{+}$, form an orthonormal basis for $\mathbf{H}_{f_{1} / f_{2}}^{2}\left(\mathbb{C}_{+}\right), \frac{f_{1}}{f_{2}}\left(f_{2} e^{i y \cdot}\right)$ can be approximated arbitrarily close by the functions in $\operatorname{span}\left\{\frac{f_{1}}{f_{2}} e_{j}: j \in \mathbb{Z}_{+}\right\}$in the Hilbert space $\mathbf{H}^{2}\left(\mathbb{C}_{+}\right)$. This fact reveals that $f_{1} e^{i y .}$ is contained in the closure of $\operatorname{span}\left\{\frac{f_{1}}{f_{2}} e_{j}: j \in \mathbb{Z}_{+}\right\}$in $\mathbf{H}^{2}\left(\mathbb{C}_{+}\right)$. Noting that $f_{1}$ is an outer function completes the proof.

To give a concrete example for Theorem 3.2, we fix $a \in \mathbb{C}_{+}$and introduce

$$
f_{1}(z):=\frac{\sqrt{1-|K(a)|^{2}}}{1+\overline{K(a)}} \frac{1}{z-\bar{a}}, \quad f_{2}(z):=\frac{1}{1-i z}, \quad z \in \mathbb{C}_{+} .
$$

Clearly, $f_{1}, f_{2}$ belong to $\mathbf{H}^{2}\left(\mathbb{C}_{+}\right)$. One may use the following Jensen formula (see, [22], page 59) to verify if an analytic function is an outer function. 
Lemma 3.3 Set $0<r<R$. If $g$ is analytic in $\{z \in \mathbb{C}:|z|<R\}, g(0) \neq 0$ and $z_{j}$, $j \in \mathbb{N}_{n}$, are the zeros of $g$ in $\{z \in \mathbb{C}:|z| \leq r\}$ then there holds

$$
\frac{1}{2 \pi} \int_{-\pi}^{\pi} \log \left|g\left(r e^{i \theta}\right)\right| d \theta=\log |g(0)|+\sum_{k \in \mathbb{N}_{n}} \log \left(\frac{r}{\left|z_{k}\right|}\right) .
$$

If analytic function $g$ in $\{z \in \mathbb{C}:|z|<R\}$ has no zeros in $\{z \in \mathbb{C}:|z| \leq r\}$ then there holds

$$
\frac{1}{2 \pi} \int_{-\pi}^{\pi} \log \left|g\left(r e^{i \theta}\right)\right| d \theta=\log |g(0)|
$$

Proposition 3.4 The function $f_{1}$ given in (3.9) is an outer function in $\mathbf{H}^{2}\left(\mathbb{C}_{+}\right)$.

Proof It suffices to show that $(z-\bar{a})^{-1}$ is an outer function. For this purpose, we calculate using the change of variables (2.4) to get that

$$
\begin{aligned}
\frac{1}{\pi} \int_{\mathbb{R}} \frac{1}{1+t^{2}} \log \left|\frac{1}{t-\bar{a}}\right| \mathrm{d} t= & -\frac{1}{2 \pi} \int_{-\pi}^{\pi} \log \left|(i-\bar{a})-(i+\bar{a}) e^{i s}\right| d s \\
& +\frac{1}{2 \pi} \int_{-\pi}^{\pi} \log \left|1+e^{i s}\right| d s .
\end{aligned}
$$

By Lemma 3.3, the first summand in the right hand side of the above equation is equal to $-\log |i-\bar{a}|$. The second summand vanishes as shown by the calculus of residues (see, [1], page 160). It is concluded by (3.7) that $f_{1}$ is an outer function.

Moreover, there holds the estimate

$$
\left|\frac{f_{1}(z)}{f_{2}(z)}\right|^{2} \leq \frac{1+|K(a)|}{1-|K(a)|}, \quad z \in \mathbb{C}_{+} .
$$

Our third step is to construct an orthonormal basis for $\mathbf{H}_{f_{1} / f_{2}}^{2}\left(\mathbb{C}_{+}\right)$. This is done in the next proposition.

\section{Proposition 3.5 The functions}

$$
e_{j}(z):=\frac{1}{\sqrt{\pi}}\left(\frac{\bar{a}-i}{a+i}\right)^{j}\left(\frac{z-a}{z-\bar{a}}\right)^{j} \frac{1}{1-i z}, \quad z \in \mathbb{C}_{+}, \quad j \in \mathbb{Z}_{+},
$$

form an orthonormal basis for $\mathbf{H}_{f_{1} / f_{2}}^{2}\left(\mathbb{C}_{+}\right)$.

Proof Note that $e_{j} \in \mathbf{H}^{2}\left(\mathbb{C}_{+}\right), j \in \mathbb{Z}_{+}$. By the isomorphism (2.5), we have for each $j, k \in \mathbb{Z}_{+}$that

$$
\left\langle e_{j}, e_{k}\right\rangle_{\mathbf{H}_{f_{1} / f_{2}}^{2}\left(\mathbb{C}_{+}\right)}=\left\langle e_{j} \frac{f_{1}}{f_{2}}, e_{k} \frac{f_{1}}{f_{2}}\right\rangle_{\mathbf{H}^{2}\left(\mathbb{C}_{+}\right)}=\left\langle T^{-1}\left(e_{j} \frac{f_{1}}{f_{2}}\right), T^{-1}\left(e_{k} \frac{f_{1}}{f_{2}}\right)\right\rangle_{\mathbf{H}^{2}(\mathbb{U})} .
$$


Set $b:=K(a)$. We get by (3.11) that

$$
\left\langle e_{j}, e_{k}\right\rangle_{\mathbf{H}_{f_{1} / f_{2}}^{2}\left(\mathbb{C}_{+}\right)}=\frac{1}{2 \pi} \int_{-\pi}^{\pi}\left(\frac{e^{i t}-b}{1-\bar{b} e^{i t}}\right)^{j-k} \frac{1-|b|^{2}}{\left|1-\bar{b} e^{i t}\right|^{2}} \mathrm{~d} t .
$$

Define

$$
m(z):=\frac{z-b}{1-\bar{b} z}, \quad z \in \mathbb{U} .
$$

Applying the change of variables $e^{i s}=m\left(e^{i t}\right)$ to the integral in the right hand side of (3.12) yields that

$$
\left\langle e_{j}, e_{k}\right\rangle_{\mathbf{H}_{f_{1} / f_{2}}^{2}\left(\mathbb{C}_{+}\right)}=\frac{1}{2 \pi} \int_{-\pi}^{\pi} e^{i(j-k) s} d s=\delta_{j, k} .
$$

We conclude that $e_{j}, j \in \mathbb{Z}_{+}$, are orthonormal in $\mathbf{H}_{f_{1} / f_{2}}^{2}\left(\mathbb{C}_{+}\right)$. To complete the proof, it suffices to show that their linear span is dense in $\mathbf{H}_{f_{1} / f_{2}}^{2}\left(\mathbb{C}_{+}\right)$. Suppose that $f \in \mathbf{H}_{f_{1} / f_{2}}^{2}\left(\mathbb{C}_{+}\right)$is orthogonal to $e_{j}$ in $\mathbf{H}_{f_{1} / f_{2}}^{2}\left(\mathbb{C}_{+}\right)$for all $j \in \mathbb{Z}_{+}$. Then similar arguments as those engaged to obtain (3.11) are able to prove that

$$
g(z):=\left[T^{-1}\left(f \frac{f_{1}}{f_{2}}\right)\right] \circ m^{-1}(z), z \in \mathbb{U}
$$

is orthogonal to $z^{j}$ in $\mathbf{H}^{2}(\mathbb{U})$ for all $j \in \mathbb{Z}_{+}$. Therefore, $f$ is a trivial function.

By Theorem 3.2, (3.10) and Propositions 3.4, 3.5,

$$
\frac{1}{\sqrt{\pi}} \frac{\sqrt{1-|K(a)|^{2}}}{1+\overline{K(a)}}\left(\frac{\bar{a}-i}{a+i}\right)^{j}\left(\frac{z-a}{z-\bar{a}}\right)^{j} \frac{1}{z-\bar{a}}, \quad j \in \mathbb{Z}_{+}
$$

form an orthonormal basis for $\mathbf{H}^{2}\left(\mathbb{C}_{+}\right)$. We shall transform it into one for $L_{\mathrm{r}}^{2}(\mathbb{R})$ by Theorem 3.1. Set $a_{r}:=\operatorname{Re}(a), a_{i}:=\operatorname{Im}(a), b:=K(a)$ and $\mu \in \partial \mathbb{U}$ such that

$$
v:=\frac{\mu}{\sqrt{\pi}} \frac{\sqrt{1-|K(a)|^{2}}}{1+\overline{K(a)}}>0 .
$$

We also denote for each $\lambda \in \mathbb{U}$ by $\zeta_{\lambda}$ the real function on $[-\pi, \pi]$ defined by

$$
\frac{e^{i s}-\lambda}{1-\bar{\lambda} e^{i s}}=e^{i \zeta_{\lambda}(s)}, \quad s \in[-\pi, \pi] \text {. }
$$

It has a positive derivative as shown explicitly below

$$
\zeta_{\lambda}^{\prime}(s)=\frac{1-|\lambda|^{2}}{1-2 \operatorname{Re}\left(\lambda e^{-i s}\right)+|\lambda|^{2}}, \quad s \in(-\pi, \pi) .
$$

Multiplying each of the functions (3.13) by the constant $\mu i$ and calculating the nontangential boundary limits of the resulting new functions yields that for amplitudes and phases given by

$$
\gamma_{j}(t):=\frac{v}{\sqrt{\left(t-a_{r}\right)^{2}+a_{i}^{2}}}, \theta_{j}(t):=j \zeta_{b}(2 \arctan t)+\arctan \frac{t-a_{r}}{a_{i}}, \quad t \in \mathbb{R}, \quad j \in \mathbb{Z}_{+},
$$


functions

$$
\begin{aligned}
\left(\gamma_{j} \cos \theta_{j}\right)(t)= & \frac{v a_{i}}{\left(t-a_{r}\right)^{2}+a_{i}^{2}} \cos \left(j \zeta_{b}(2 \arctan t)\right) \\
& +\frac{v\left(a_{r}-t\right)}{\left(t-a_{r}\right)^{2}+a_{i}^{2}} \sin \left(j \zeta_{b}(2 \arctan t)\right), \quad t \in \mathbb{R}, \quad j \in \mathbb{Z}_{+}
\end{aligned}
$$

and

$$
\begin{aligned}
\left(\gamma_{j} \sin \theta_{j}\right)(t)= & \frac{v\left(t-a_{r}\right)}{\left(t-a_{r}\right)^{2}+a_{i}^{2}} \cos \left(j \zeta_{b}(2 \arctan t)\right) \\
& +\frac{v a_{i}}{\left(t-a_{r}\right)^{2}+a_{i}^{2}} \sin \left(j \zeta_{b}(2 \arctan t)\right), t \in \mathbb{R}, \quad j \in \mathbb{Z}_{+}
\end{aligned}
$$

form an orthonormal basis for $L_{\mathrm{r}}^{2}(\mathbb{R})$ that satisfies (3.2) and $\theta_{j}^{\prime}>0, j \in \mathbb{Z}_{+}$.

\section{A second construction}

Our second construction is stimulated by the following simple observation.

Lemma 4.1 If $g$ is a function in $\mathbf{H}^{\infty}\left(\mathbb{C}_{+}\right)$then

$$
\frac{1}{\pi} \int_{\mathbb{R}} \frac{g(t)}{1+t^{2}} \frac{i-t}{i+t} d t=0 .
$$

Proof By the change of variables (2.4), we see that

$$
\frac{1}{\pi} \int_{\mathbb{R}} \frac{g(t)}{1+t^{2}} \frac{i-t}{i+t} \mathrm{~d} t=\frac{1}{2 \pi} \int_{-\pi}^{\pi} e^{i s}\left(g \circ K^{-1}\right)\left(e^{i s}\right) d s=\frac{1}{2 \pi i} \int_{\partial \mathbb{U}}\left(g \circ K^{-1}\right)(z) d z .
$$

By the Cauchy integral formula (2.10), we have for each $r \in(0,1)$ that

$$
\frac{1}{2 \pi i} \int_{\partial \mathbb{U}}\left(g \circ K^{-1}\right)(r z) d z=0 .
$$

Since $g \circ K^{-1} \in \mathbf{H}^{\infty}(\mathbb{U}),\left(g \circ K^{-1}\right)(r \cdot)$ converges in $L^{1}(\partial \mathbb{U})$ to $g \circ K^{-1}$ as $r$ goes to 1 (see, [18], page 340). This fact together with (4.3) proves that the last integral in (4.2) vanishes and hence completes the proof.

The finite Blaschke product associated with a finite number of points $z_{j} \in$ $\mathbb{C}_{+}, j \in \mathbb{N}_{n}$, is the analytic function $f$ on $\mathbb{C}_{+}$defined as

$$
f(z):=\prod_{j \in \mathbb{N}_{n}} \frac{z-z_{j}}{z-\overline{z_{j}}}, \quad z \in \mathbb{C}_{+} .
$$


Suppose we have a sequence of functions $f_{n} \in \mathbf{H}^{\infty}\left(\mathbb{C}_{+}\right), n \in \mathbb{N}$, with the properties that $f_{n}(i)=0$ and

$$
\overline{f_{n}(t)}=\left(\frac{h_{n}}{g_{n}}\right)(t), \quad t \in \mathbb{R}, \quad n \in \mathbb{N},
$$

where $h_{n}, g_{n}$ are analytic functions on $\mathbb{C}_{+}$with $g_{n}$ having at least one but a finite number of zeros in $\mathbb{C}_{+}$. Let $b_{n}$ be the finite Blaschke product associated with the zeros of $g_{n}$ in $\mathbb{C}_{+}, n \in \mathbb{N}$. With such a sequence of analytic functions, we define

$$
\beta_{0}(z):=\frac{1}{\sqrt{\pi}} \frac{1}{1-i z}, \quad \beta_{n}(z):=\frac{1}{\sqrt{\pi}} \frac{1}{1-i z} f_{n}(z) \prod_{j \in \mathbb{N}_{n-1}} b_{j}(z), \quad z \in \mathbb{C}_{+}, n \in \mathbb{N} .
$$

Here we denote $\mathbb{N}_{0}:=\emptyset$.

Theorem 4.2 The functions $\beta_{n}, n \in \mathbb{Z}_{+}$, constructed by (4.5) are orthogonal in $\mathbf{H}^{2}\left(\mathbb{C}_{+}\right)$.

Proof We observe that $\beta_{n} \in \mathbf{H}^{2}\left(\mathbb{C}_{+}\right), n \in \mathbb{N}$, because they are products of a function in $\mathbf{H}^{2}\left(\mathbb{C}_{+}\right)$and a bounded analytic function. For $n \in \mathbb{N}$, we have that

$$
\left\langle\beta_{n}, \beta_{0}\right\rangle_{\mathbf{H}^{2}\left(\mathbb{C}_{+}\right)}=\frac{1}{\pi} \int_{\mathbb{R}} \frac{1}{1+t^{2}} f_{n}(t) \prod_{j \in \mathbb{N}_{n-1}} b_{j}(t) \mathrm{d} t,
$$

which, by Lemma 4.1, equals to zero since

$$
f_{n}(z)=(i+z) \frac{f_{n}(z)}{i-z} \frac{i-z}{i+z}, z \in \mathbb{C}_{+}
$$

with $(i+z) \frac{f_{n}}{i-z}$ being bounded and analytic on $\mathbb{C}_{+}$. It is also calculated for $n>m \geq 1$ by (4.4) that

$$
\begin{aligned}
\left\langle\beta_{n}, \beta_{m}\right\rangle_{\mathbf{H}^{2}\left(\mathbb{C}_{+}\right)} & =\int_{\mathbb{R}} \beta_{n}(t) \overline{\beta_{m}(t)} \mathrm{d} t \\
& =\frac{1}{\pi} \int_{\mathbb{R}} \frac{1}{1+t^{2}} f_{n}(t)\left(\frac{h_{m}}{g_{m}}\right)(t)\left(\prod_{j=m}^{n-1} b_{j}(t)\right) \mathrm{d} t \\
& =\frac{1}{\pi} \int_{\mathbb{R}} \frac{1}{1+t^{2}} f_{n}(t)\left(h_{m} \frac{b_{m}}{g_{m}}\right)(t)\left(\prod_{j=m+1}^{n-1} b_{j}(t)\right) \mathrm{d} t .
\end{aligned}
$$

The integral above is also equal to zero since the function

$$
f_{n} h_{m} \frac{b_{m}}{g_{m}} \prod_{j=m+1}^{n-1} b_{j}
$$

is bounded and analytic on $\mathbb{C}_{+}$with a zero at $z=i$. 
Let us see a simplest example of the above construction. In this example, $h_{n}$ in (4.4) is only to meet the requirement that $f_{n}(i)=0$ and $g_{n}$ has a single zero in $\mathbb{C}_{+}$. In other words, we set

$$
h_{n}(z):=z+i, \quad g_{n}(z):=z-d_{n}, \quad z \in \mathbb{C}_{+}, \quad n \in \mathbb{N},
$$

where $d_{n} \in \mathbb{C}_{+}, n \in \mathbb{N}$. As a consequence, we have for $z \in \mathbb{C}_{+}$that

$$
b_{n}(z):=\frac{z-d_{n}}{z-\overline{d_{n}}}
$$

and

$$
\beta_{0}(z):=\frac{1}{\sqrt{\pi}} \frac{1}{1-i z}, \quad \beta_{n}(z):=\frac{1}{\sqrt{\pi}} \frac{1}{1-i z} \frac{z-i}{z-\overline{d_{n}}} \prod_{j \in \mathbb{N}_{n-1}} \frac{z-d_{j}}{z-\overline{d_{j}}}, \quad n \in \mathbb{N} .
$$

It can be verified directly that the phases of the above functions also possess nonnegative derivatives.

Theorem 4.3 Let $\beta_{j}, j \in \mathbb{Z}_{+}$, be constructed as in (4.6) where $d_{n} \in \mathbb{C}_{+}, n \in \mathbb{N}$, are pairwise distinct. Then $\operatorname{span}\left\{\beta_{j}: j \in \mathbb{Z}_{+}\right\}$is dense in $\mathbf{H}^{2}\left(\mathbb{C}_{+}\right)$if and only if

$$
\sum_{n \in \mathbb{N}}\left(1-\left|K\left(d_{n}\right)\right|\right)=\infty
$$

Proof Let $\varphi \in \mathbf{H}^{2}\left(\mathbb{C}_{+}\right)$. Using the isomorphism (2.5), one can show by induction that $\varphi$ is orthogonal to $\beta_{j}$ for all $j \in \mathbb{Z}_{+}$if and only if

$$
\varphi(i)=0, \varphi\left(d_{n}\right)=0, \quad n \in \mathbb{N} .
$$

It hence suffices to point out the fact that there does not exist a nontrivial function in $\mathbf{H}^{2}\left(\mathbb{C}_{+}\right)$that vanishes on $\left\{d_{n}: n \in \mathbb{N}\right\}$ if and only if (4.7) holds, [9].

We now use Theorems 4.2 and 4.3 to derive orthonormal bases for $L_{\mathrm{r}}^{2}(\mathbb{R})$. Choose pairwise distinct $d_{n} \in \mathbb{C}_{+}, n \in \mathbb{N}$, that satisfy (4.7). This can be done by, for example, requiring that $\left|K\left(d_{n}\right)\right|=1-n^{-1}, n \in \mathbb{N}$. Set $d_{n, r}:=\operatorname{Re}\left(d_{n}\right)$, $d_{n, i}:=\operatorname{Im}\left(d_{n}\right)$ and $b_{n}:=K\left(d_{n}\right), n \in \mathbb{N}$. We modify the construction (4.6) slightly to get that

$$
\frac{1}{\sqrt{\pi}} \frac{1}{1-i z}, \quad \sqrt{\frac{d_{n, i}}{\pi}} \frac{i-z}{z+i} \frac{i}{z-\overline{d_{n}}} \prod_{j \in \mathbb{N}_{n-1}}\left(\frac{z-d_{j}}{z-\overline{d_{j}}} \frac{\overline{d_{j}}-i}{d_{j}+i}\right), n \in \mathbb{N},
$$

constitute an orthonormal bases for $\mathbf{H}^{2}\left(\mathbb{C}_{+}\right)$. As discussed in the example at the end of the last section, the phase of each of the basis functions has a positive 
derivative. Finally, we calculate the nontangential boundary limits of the basis functions to conclude that the following functions

$$
\begin{gathered}
\frac{1}{\sqrt{\pi}} \frac{1}{1+t^{2}}, \frac{1}{\sqrt{\pi}} \frac{t}{1+t^{2}} \\
\sqrt{\frac{d_{n, i}}{\pi}} \frac{d_{n, i}}{\left(t-d_{n, r}\right)^{2}+d_{n, i}^{2}} \cos \left(\omega_{n}(2 \arctan t)\right) \\
+\sqrt{\frac{d_{n, i}}{\pi}} \frac{d_{n, r}-t}{\left(t-d_{n, r}\right)^{2}+d_{n, i}^{2}} \sin \left(\omega_{n}(2 \arctan t)\right), n \in \mathbb{N}
\end{gathered}
$$

and

$$
\begin{aligned}
& \sqrt{\frac{d_{n, i}}{\pi}} \frac{t-d_{n, r}}{\left(t-d_{n, r}\right)^{2}+d_{n, i}^{2}} \cos \left(\omega_{n}(2 \arctan t)\right) \\
& \quad+\sqrt{\frac{d_{n, i}}{\pi}} \frac{d_{n, i}}{\left(t-d_{n, r}\right)^{2}+d_{n, i}^{2}} \sin \left(\omega_{n}(2 \arctan t)\right), n \in \mathbb{N},
\end{aligned}
$$

form an orthonormal basis for $L_{\mathrm{r}}^{2}(\mathbb{R})$, where $\omega_{n}:=\zeta_{0}+\sum_{j \in \mathbb{N}_{n-1}} \zeta_{b_{j}}, n \in \mathbb{N}$.

\section{Orthonormal bases for $L_{\mathrm{r}}^{2}[-\pi, \pi]$}

In this section, we present parallel results for the construction of orthonormal bases for $L_{\mathrm{r}}^{2}[-\pi, \pi]$. We omit the proofs since their arguments are similar to those in the last two sections.

Theorem 5.1 Functions $1, m_{j}, j \in \mathbb{N}$, with nontangential boundary limits

$$
m_{j}\left(e^{i t}\right)=\rho_{j}(t) e^{i \theta_{j}(t)}, \quad t \in[-\pi, \pi], \quad j \in \mathbb{N},
$$

form an orthonormal basis for $\mathbf{H}^{2}(\mathbb{U})$ if and only if $\frac{1}{\sqrt{2 \pi}}, \frac{1}{\sqrt{\pi}} \rho_{j} \cos \theta_{j}$, $\frac{1}{\sqrt{\pi}} \rho_{j} \sin \theta_{j}, j \in \mathbb{N}$, satisfy for each $j \in \mathbb{N}$

$$
\tilde{H}\left(\rho_{j}(\cdot) \cos \theta_{j}(\cdot)\right)(t)=\rho_{j}(t) \sin \theta_{j}(t), \quad t \in[-\pi, \pi] .
$$

and constitute an orthonormal basis for $L_{r}^{2}[-\pi, \pi]$.

Our next result parallels to Theorem 3.2 in Section 3. We call $h \in \mathbf{H}(\mathbb{U})$ an outer function if it is of the form

$$
h(z)=c \exp \left\{\frac{1}{2 \pi} \int_{-\pi}^{\pi} \frac{e^{i t}+z}{e^{i t}-z}(\log \psi)\left(e^{i t}\right) \mathrm{d} t\right\}, \quad z \in \mathbb{U},
$$

where $c \in \partial \mathbb{U}, \psi$ is a positive Lebesgue measurable function on $\partial \mathbb{U}$ such that $\log \psi \in L^{1}(\partial \mathbb{U})$, [18]. According to $[3,9,18]$, a function $f \in \mathbf{H}(\mathbb{U})$ is an outer 
function if and only if the linear span of $\left\{f p_{j}: j \in \mathbb{Z}_{+}\right\}$is dense in $\mathbf{H}^{2}(\mathbb{U})$, where $p_{j}(z):=z^{j}$. We denote by $\mathbf{H}_{f}^{2}(\mathbb{U})$ for an $f \in \mathbf{H}^{\infty}(\mathbb{U})$ the Hilbert space completed upon the linear space of functions in $\mathbf{H}^{2}(\mathbb{U})$ endowed with the following inner product

$$
\langle g, h\rangle_{\mathbf{H}_{f}^{2}(\mathbb{U})}:=\frac{1}{2 \pi} \int_{-\pi}^{\pi} g\left(e^{i t}\right) \overline{h\left(e^{i t}\right)}\left|f\left(e^{i t}\right)\right|^{2} \mathrm{~d} t, \quad g, h \in \mathbf{H}_{f}^{2}(\mathbb{U}) .
$$

Theorem 5.2 Let $f \in \mathbf{H}^{2}(\mathbb{U})$ be a bounded outer function. If $e_{j} \in \mathbf{H}^{2}(\mathbb{U}), j \in$ $\mathbb{Z}_{+}$, form an orthonormal basis for $\mathbf{H}_{f}^{2}(\mathbb{U})$ then $f e_{j}, j \in \mathbb{Z}_{+}$, constitute an orthonormal basis for $\mathbf{H}^{2}(\mathbb{U})$.

We also have a construction similar to the one in Section 4. A finite Blaschke product $f \in \mathbf{H}^{2}(\mathbb{U})$ associated with $\left\{z_{j}: j \in \mathbb{N}_{n}\right\} \subseteq \mathbb{U}$ is the function

$$
f(z):=\prod_{j \in \mathbb{N}_{n}} \frac{z-z_{j}}{1-\overline{z_{j}} z}, \quad z \in \mathbb{U} .
$$

Theorem 5.3 Suppose that $f_{n}, n \in \mathbb{N}$, is a sequence of functions in $\mathbf{H}^{\infty}(\mathbb{U})$ with the properties that for each $n \in \mathbb{N}, f_{n}(0)=0$ and there exist analytic functions $h_{n}, g_{n}$ on $\mathbb{U}$ such that

$$
\overline{f_{n}\left(e^{i t}\right)}=\left(\frac{h_{n}}{g_{n}}\right)\left(e^{i t}\right), \quad t \in[-\pi, \pi]
$$

and $g_{n}$ has at least one but a finite number of zeros in $\mathbb{U}$. Then

$$
m_{n}(z):=f_{n}(z) \prod_{j \in \mathbb{N}_{n-1}} b_{j}(z), \quad z \in \mathbb{U}, n \in \mathbb{N},
$$

are orthogonal in $\mathbf{H}^{2}(\mathbb{U})$, where $b_{n}$ is the finite Blaschke product associated with the zeros of $g_{n}$.

Particular examples of bases for $L_{\mathrm{r}}^{2}[-\pi, \pi]$ that fit into the general constructions described by the last two theorems can be found in ([5] and Wang et al., preprint).

\section{References}

1. Ahlfors, L.V.: Complex Analysis, 3rd edn. McGraw-Hill, New York (1979)

2. Bedrosian, E.: A product theorem for Hilbert transforms. Proc. IEEE 51, 868-869 (1963)

3. Beurling, A.: On two problems concerning linear transformations in Hilbert space. Acta Math. 81, 239-255 (1949)

4. Chen, Q., Huang, N.E., Riemenschneider, S., Xu, Y.: A B-spline approach for empirical mode decompositions. Adv. Comput. Math. 24, 171-195 (2006)

5. Chen, Q., Li, L., Qian, T.: Two families of unit analytic signals with non-linear phase. Phys. D 221, 1-12 (2006)

6. Cohen, L.: Time-Frequency Analysis: Theory and Applications. Prentice Hall, New Jersey (1995) 
7. Conway, J.B.: A Course in Functional Analysis, 2nd edn. Springer, New York (1990)

8. Duren, P.L.: Theory of $H^{p}$ Spaces. Academic, New York (1970)

9. Garnett, J.B.: Bounded Analytic Functions. Academic, New York (1981)

10. Grafakos, L.: Classical and Modern Fourier Analysis. Prentice Hall, New Jersey (2004)

11. Huang, N.E., et al.: The empirical mode decomposition and the Hilbert spectrum for nonlinear and non-stationary time series analysis. Proc. Roy. Soc. London Ser. A 454, 903-995 (1998)

12. Lax, P.D.: Translation invariant spaces. Acta Math. 101, 163-178 (1959)

13. Liu, B., Riemenschneider, S., Xu, Y.: Gearbox fault diagnosis using empirical mode decomposition and Hilbert spectrum. Mech. Syst. Signal Process. 20, 718-734 (2006)

14. Liu, Y., Xu, Y.: Piecewise linear spectral sequences. Proc. Amer. Math. Soc. 133, 2297-2308 (2005)

15. Picibono, B.: On instantaneous amplitude and phase of signals. IEEE Trans. Signal Process. 45, 552-560 (1997)

16. Qian, T.: Characterization of boundary values of functions in Hardy spaces with applications in signal analysis. J. Integral Equations Appl. 17, 159-198 (2005)

17. Qian, T., Chen, Q., Li, L.: Analytic unit quadrature signals with nonlinear phase. Phys. D 203, 80-87 (2005)

18. Rudin, W.: Real and Complex Analysis, 3rd edn. McGraw-Hill, New York (1987)

19. Xu, Y., Liu, B., Liu, J., Riemenschneider, S.: Two-dimensional empirical mode decomposition by finite elements. Proc. Roy. Soc. London Ser. A 462, 3081-3096 (2006)

20. Xu, Y., Yan, D.: The Bedrosian identity for the Hilbert transform of product functions. Proc. Amer. Math. Soc. 134, 2719-2728 (2006)

21. Xu, Y., Zhang, H.: Recent mathematical developments on empirical mode decomposition. Advances in Adaptive Data Analysis (2009, in press)

22. Young, R.M.: An Introduction to Nonharmonic Fourier Series. Academic, New York (1980)

23. Yu, B., Zhang, H.: The Bedrosian identity and homogeneous semi-convolution equations. J. Integral Equations Appl. 20, 527-568 (2008) 